\title{
Triplex hybridization of siRNA with bifacial glycopolymer nucleic acid enables hepatocyte-targeted silencing
}

\author{
Xin Xia, ${ }^{2, \dagger}$ Zhun Zhou ${ }^{1, \dagger}$ Chris DeSantis, ${ }^{1}$ John J. Rossi ${ }^{2}$ and Dennis Bong ${ }^{1, *}$ \\ ${ }^{1}$ Department of Chemistry and Biochemistry, The Ohio State University, 100 W. 18th Avenue, Columbus, OH 43210 and the \\ ${ }^{2}$ Department of Molecular and Cellular Biology, Beckman Research Institute of City of Hope, Duarte, CA 91010, USA
}

${ }^{\dagger}$ These authors contributed equally to this work.

*To whom correspondence should be addressed.

\section{Table of Contents}

RNA CONTROL SEQUENCES FOR HepG2 SILENCING AND STABILITY EXPERIMENTS

Sequences and general design

SILENCING PROTOCOL AND OUTCOME WITH CONTROL SIRNA

RNA-POLYMER COMPLEX STABILITY TOWARDS NUCLEASE 


\section{RNA SEQUENCES FOR HepG2 SILENCING AND STABILITY EXPERIMENTS}

A previously validated siRNA sequence targeted to HIV-1 Tat/Rev mRNA(Zhou et al., 2008) was chosen for use as a negative control in HepG2 cells. This sequence has no host genome targeting and should elicit no

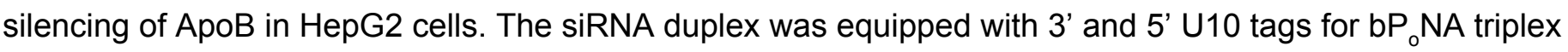
hybridization in a manner identical to the ApoB targeted siRNA. A FITC-labeled antisense sequence was also used to assess nuclease stability of the polymer complexed siRNA.

- Used for silencing as negative control:

\section{Sense:}

5'-Phos-rGrCrG rGrArG rArCrA rGrCrG rArCrG rArArG rArGrC rUrCrA rUrCrA rCrCrU rUrUrU rUrUrU rUrUrU -3'

Antisense:

5'- rUrUrU rUrUrU rUrUrU rUrCrC rUrGrA rUrGrA rGrCrU rCrUrU rCrGrU rCrGrC rUrGrU rCrUrC rCrGrC rUrU-3'

- Used for nuclease stability assays, in duplex with unmodified sense strand:

FITC-antisense

5'-FITC-rUrUrU rUrUrU rUrUrU rUrCrC rUrGrA rUrGrA rGrCrU rCrUrU rCrGrU rCrGrC rUrGrU rCrUrC rCrGrC rUrU-3' 


\section{SILENCING PROTOCOL AND OUTCOME WITH CONTROL SIRNA}

HepG2 cells were seeded in full EMEM media at the density of $2 \times 10^{5}$ cell $/ \mathrm{ml}, 0.5 \mathrm{~mL} /$ well in $24-$ well tissue culture plate. Cells were transfected with Tat/Rev-extended siRNA either by lipofectamine 2000 at $10 \mathrm{nM}$ concentration following manufacturer instructions, or via complexation with GM10 polymer at the ratio of 1:3. A concentration range from $10 \mathrm{nM}$ to $100 \mathrm{nM}$ complex (siRNA) was used for transfection. As an additional control, GM10 polymer is also transfected without siRNA complexation into HepG2 cells at the same concentrations. Cells were harvested 48 hours post-transfection, total RNA was purified using Trizol reagent, and the mRNA levels of ApoB-100 were analyzed via RT-qPCR as described in the manuscript. None of these negative control experiments resulted in ApoB silencing (Figure S1).

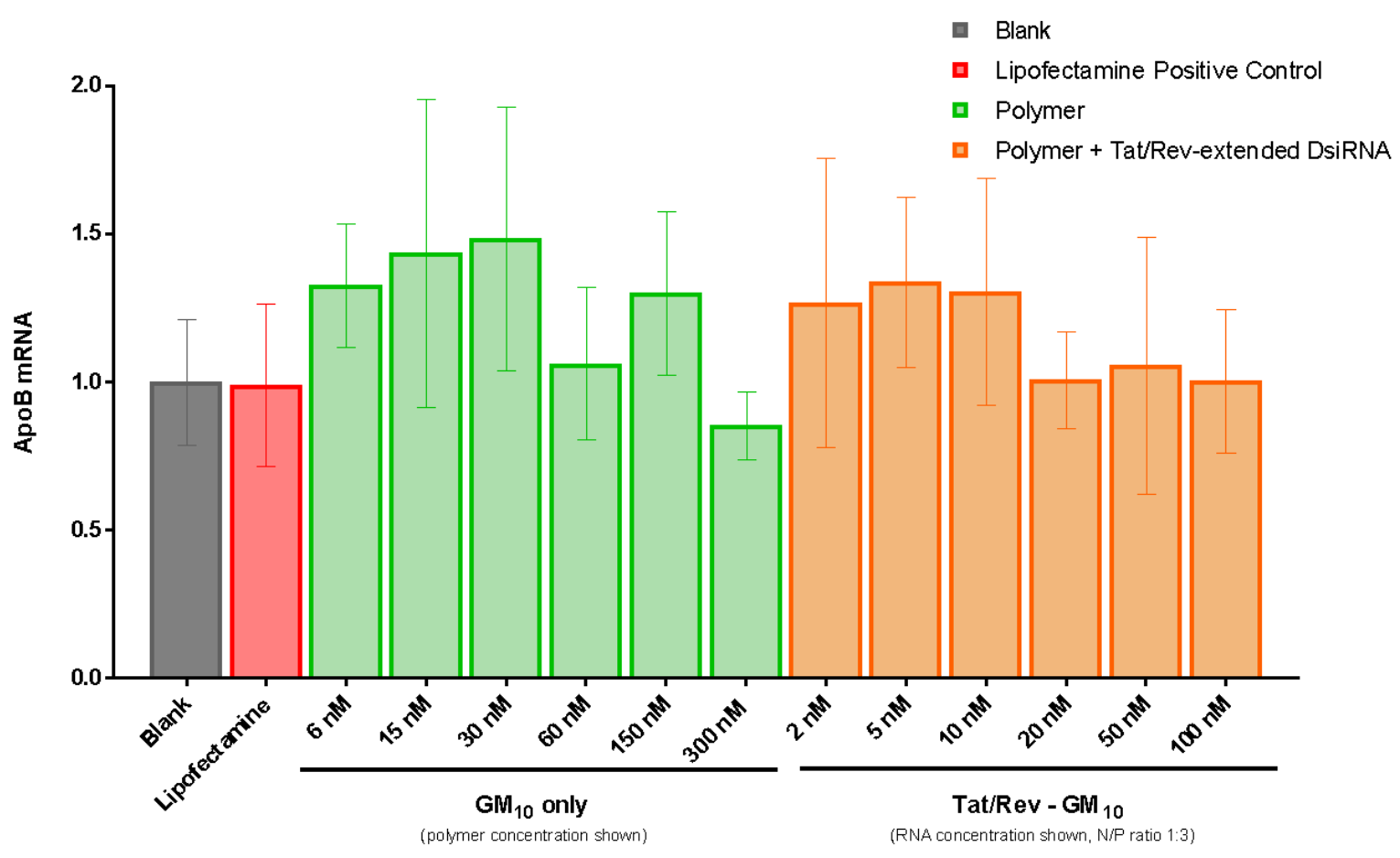

Figure S1. Relative ApoB levels in HepG2 cells from the treatment groups indicated. Error bars shown for triplicate measurements. See above protocol for experimental conditions. 


\section{RNA-POLYMER COMPLEX STABILITY TOWARDS EXONUCLEASE}

All experiments were conducted in NEBuffer 4, $\mathrm{pH} 7.9(50 \mathrm{mM}$ potassium acetate, $20 \mathrm{mM}$ Tris acetate, $10 \mathrm{mM}$ magnesium acetate, $1 \mathrm{mM}$ DTT) and supplemented with glycerol (10\%). Saturated binding conditions of GM10 polymer to Tat/Rev-extended siRNA DNA were determined by EMSA, by titrating FITC-labeled siRNA (100 nM) against GM10 polymer. Upon complexation, the fluorescence becomes diffuse due to polymer heterogeneity and formation of complexes with different electrophoretic mobility. Two bands are observed for siRNA alone, corresponding to the duplex and single-stranded form (higher mobility). Digestion by exonuclease $T$ results in coalescence to new fragment. The formation of the cleavage product is inhibited by polymer complexation over the 3 hour course of experiment. Polymer complexation towards non-modified siRNA duplex was able to protect the free ends of the siRNA from nuclease digestion for up to 3 hours, while non-complexed siRNA was readily digested to minimum stable structure within 10 minutes. Notably, similar treatment of the polymer complex with an endonuclease (Dicer) resulted in expected processing (see manuscript). A general protocol for treatment with nuclease is as follows: exonuclease $\mathrm{T}$ (20 units) was added to a reaction solution (150 $\mu \mathrm{L})$ in conditions under which complete GM10-siRNA complexation was observed and incubated at $37^{\circ} \mathrm{C}$ for $3 \mathrm{~h}$. Aliquots were quenched with EDTA (60 mM final concentration) at various time points, examined on native gradient PAGE gel (4-16\%) and the gel imaged by FITC fluorescence (Figure S2).

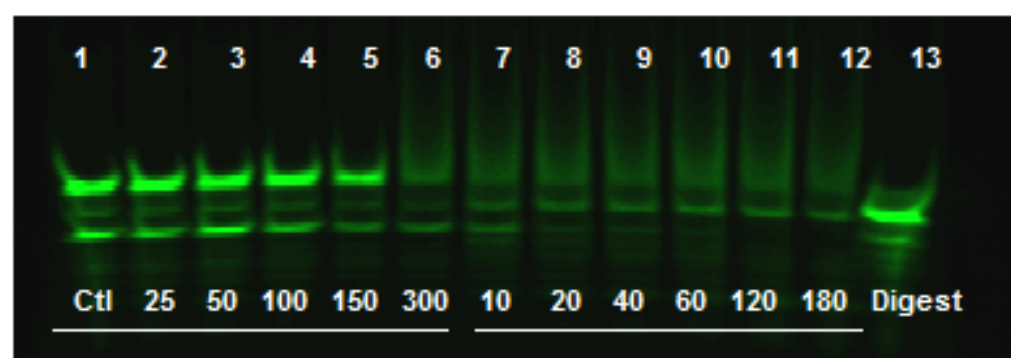

[GM10] /nM

Time / min

Figure S2. Native gel of siRNA-polymer complexes. Lanes 1-6: 100nM FITC-siRNA was incubated with indicated concentrations of GM10, leading to a full saturation at an siRNA:polymer ratio of 1:3 (lane 6). The complex from lane 6 then was subjected to Exonuclease $\mathrm{T}$ digestion at $37^{\circ} \mathrm{C}$ for 3 hours, aliquots of the reaction were quenched at specific time points with 60mM EDTA (lane 7-12). A positive digestion control was analyzed in lane 13, where $100 \mathrm{nM}$ siRNA duplex was subjected to the same concentration of ExoT for 10 minutes at $37^{\circ} \mathrm{C}$. 


\section{POLYMER SYNTHESIS PROTOCOLS AND CHARACTERIZATION}

Analytical size-exclusion chromatography was performed in THF at a flow rate of $1 \mathrm{ml} / \mathrm{min}$ with refractive index detection or UV detection (trithiocarbonate absorbance, $300 \mathrm{~nm}$ ) using four Styragel HR4 (Waters, $5 \mu \mathrm{m}$, $10-4 \AA$, $7.8 \mathrm{~mm} \times 300 \mathrm{~mm}, 5 \mathrm{~K}-600 \mathrm{~K}$ ) analytical columns in series, and number average molecular weights were determined with respect to commercially available polymethylmethacrylate standards. The use of 4 columns connected in series results in longer overall retention times.

Table S1. GPC traces of polymers

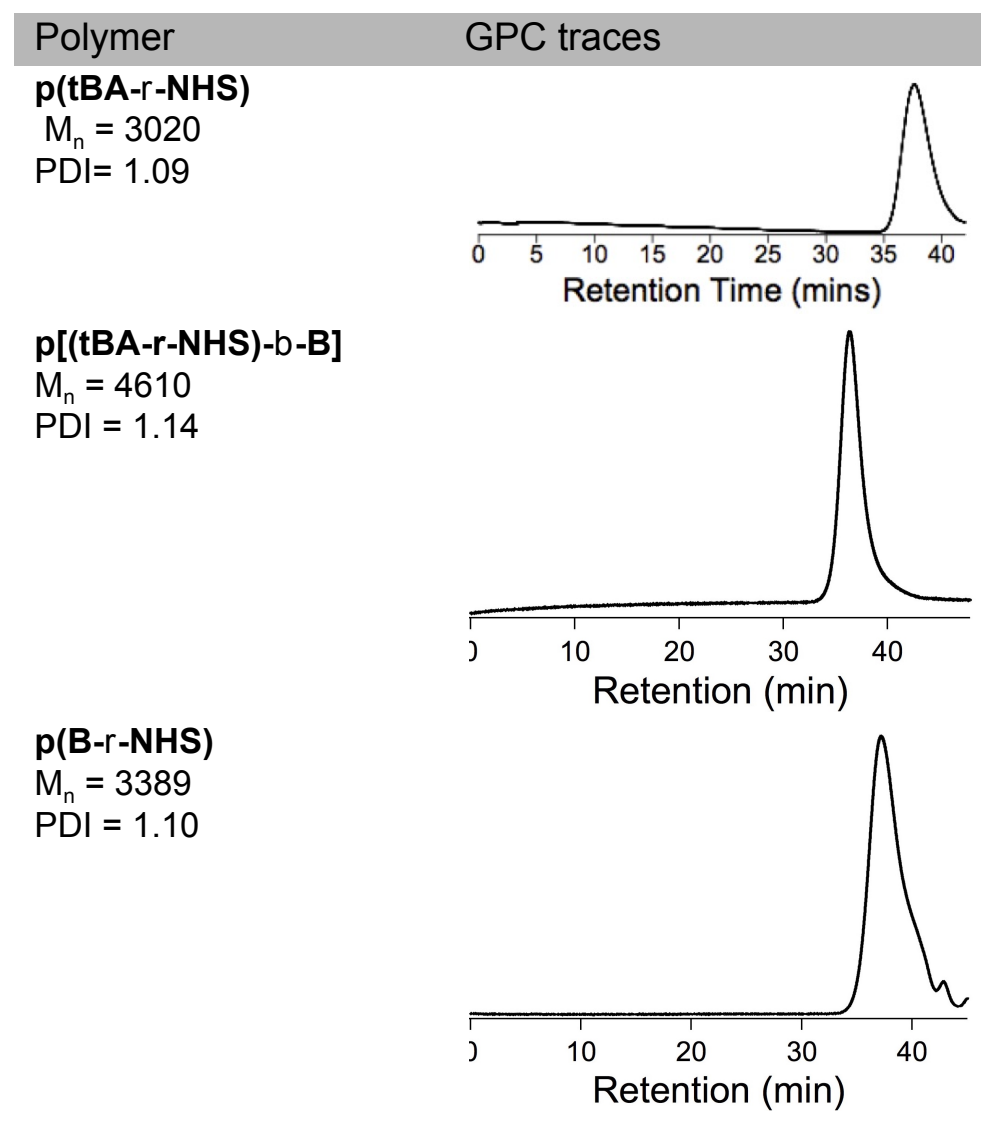




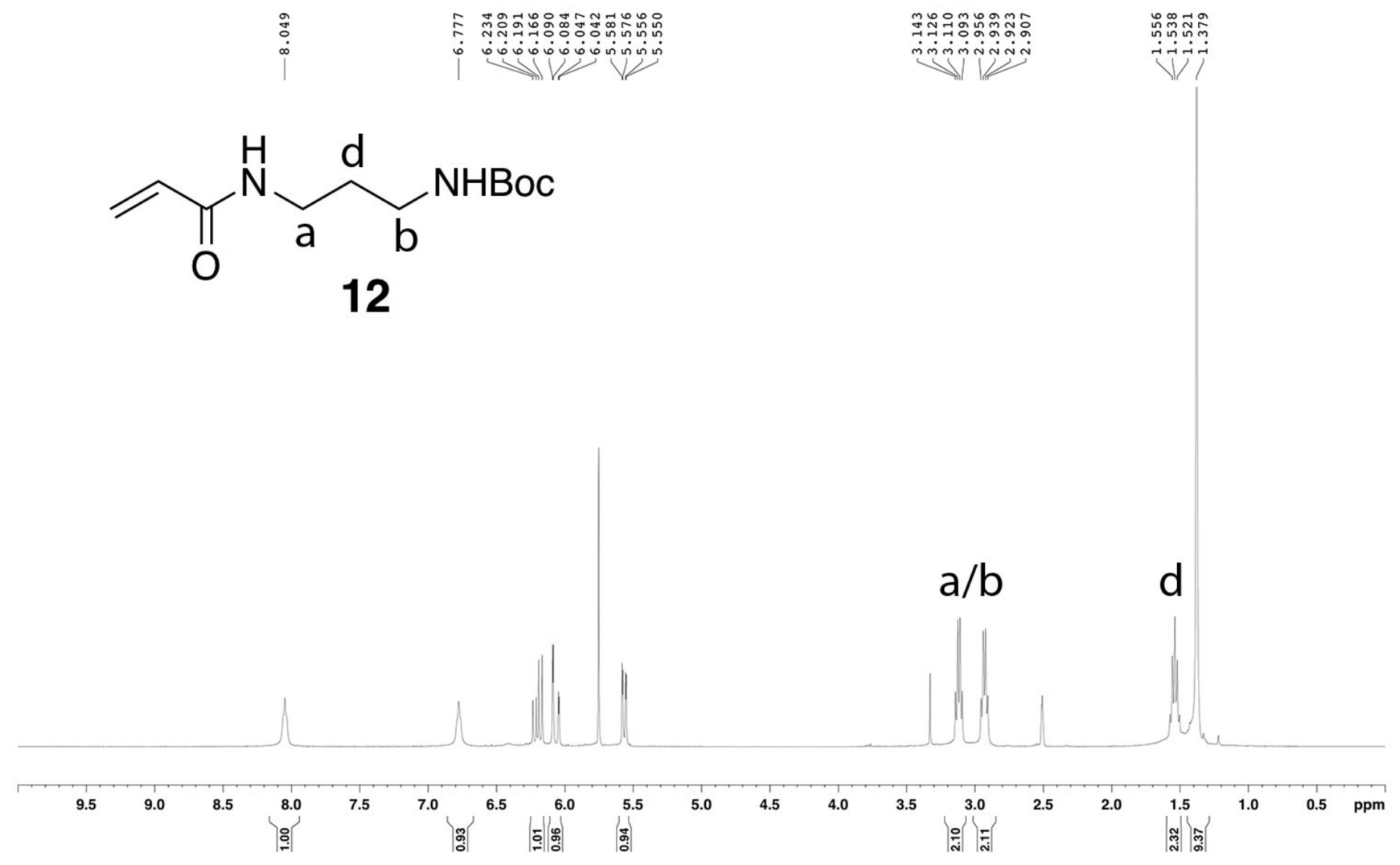

Figure S3. ${ }^{1} \mathrm{HNMR}$ of Boc-protected monomer 12, carbamic acid, N-[3-[(1-oxo-2-propen-1-yl)amino]propyl]-, 1,1-dimethylethyl ester. Spectrum matches that of the commercially available Boc-protected monomer 12. 


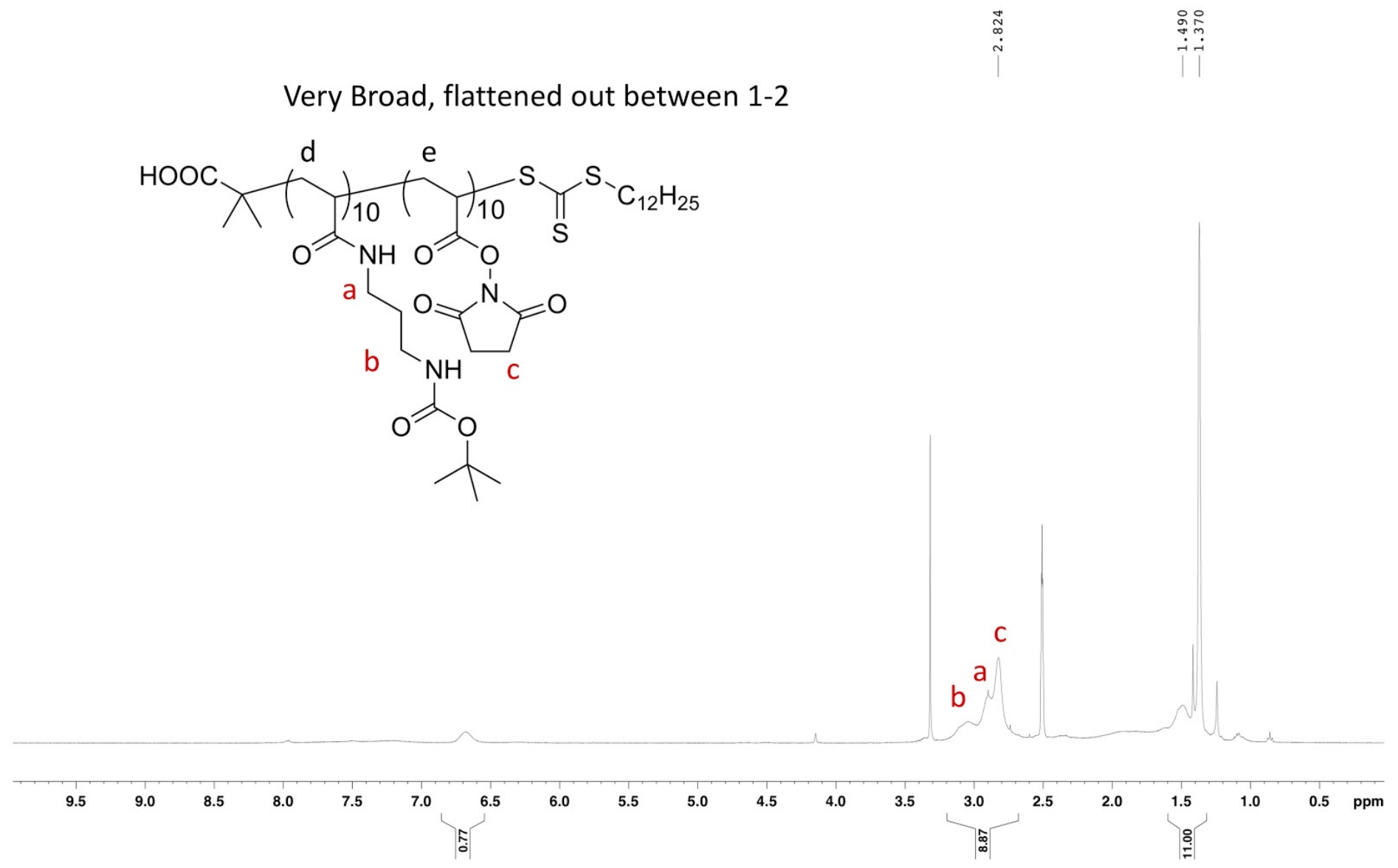

Figure S4. (NHS-r-B) $)_{8-10}$ base polymer for GM10, wherein "B" represents monomer 12 and "NHS" represents monomer 13. Resonances from both monomers $(a, b, c)$ contribute to the region from 3.2-2.6 ppm while resonance $d$ (Figure S1) and the t-butyl group contribute to the region from 1.6-1.3 ppm. Integration supports 1:1 monomer usage. 


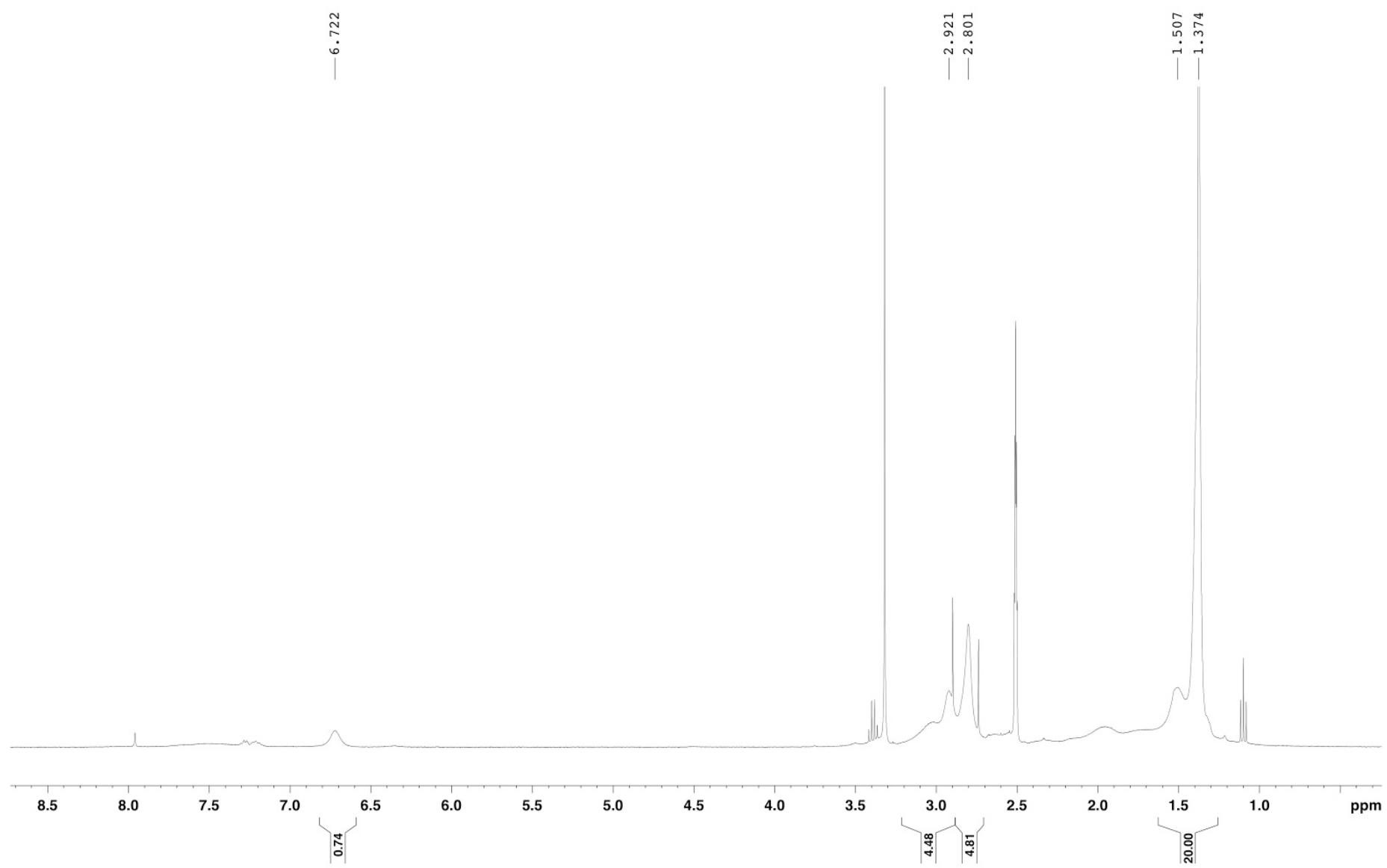

Figure S5. (tBA-r-NHS) ${ }_{10}-\mathrm{b}-\mathrm{B}_{7-10}$ diblock base polymer for AM10-G10. Assignments made as indicated above. 
$\mathrm{H}^{1}$-NMR assignment for GalNAc-NHS (DMSO-d6)
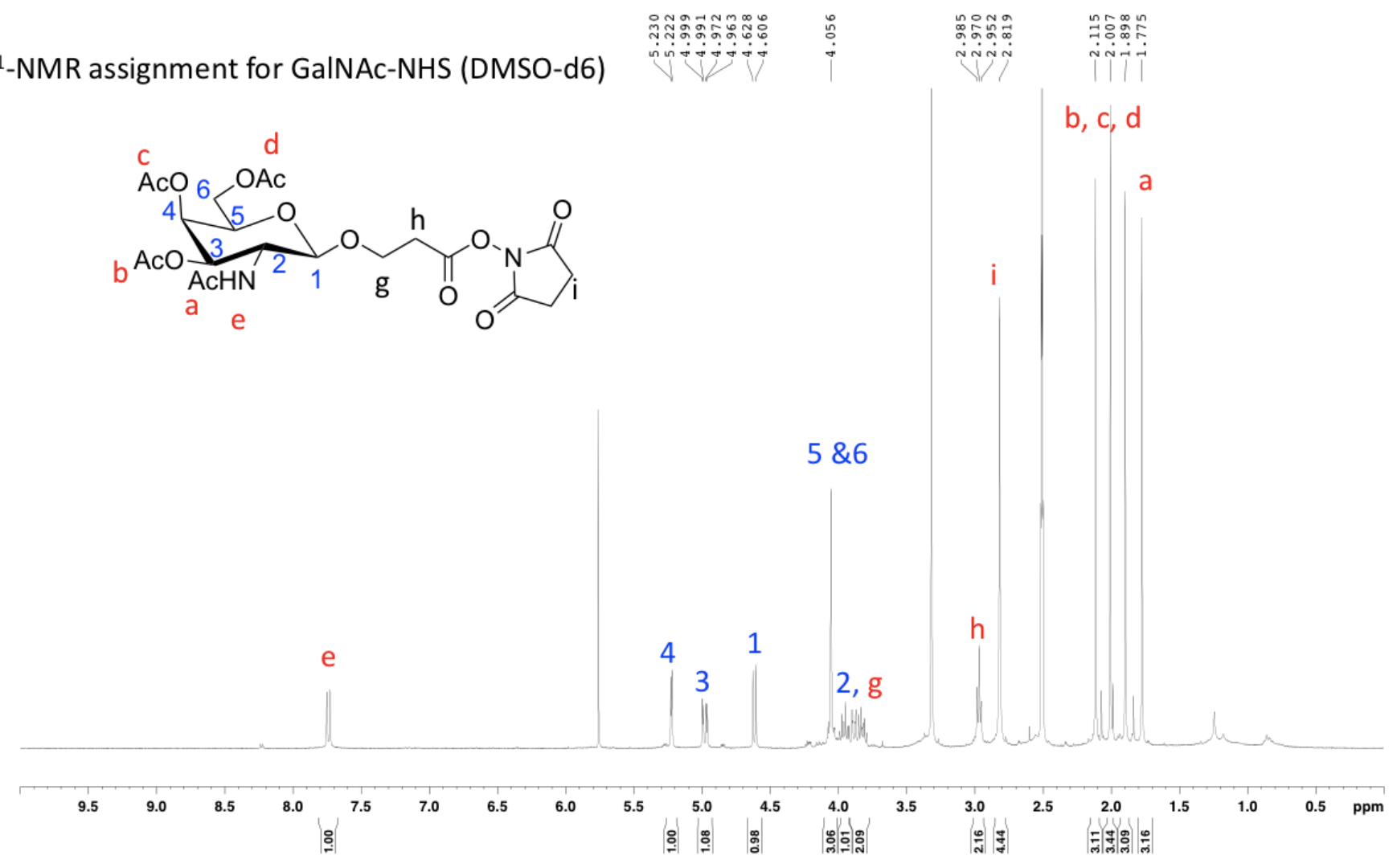

b, c, d

Figure S6. H1-NMR Spectrum of GalNAc -NHS, the assignments are made as indicated above. 

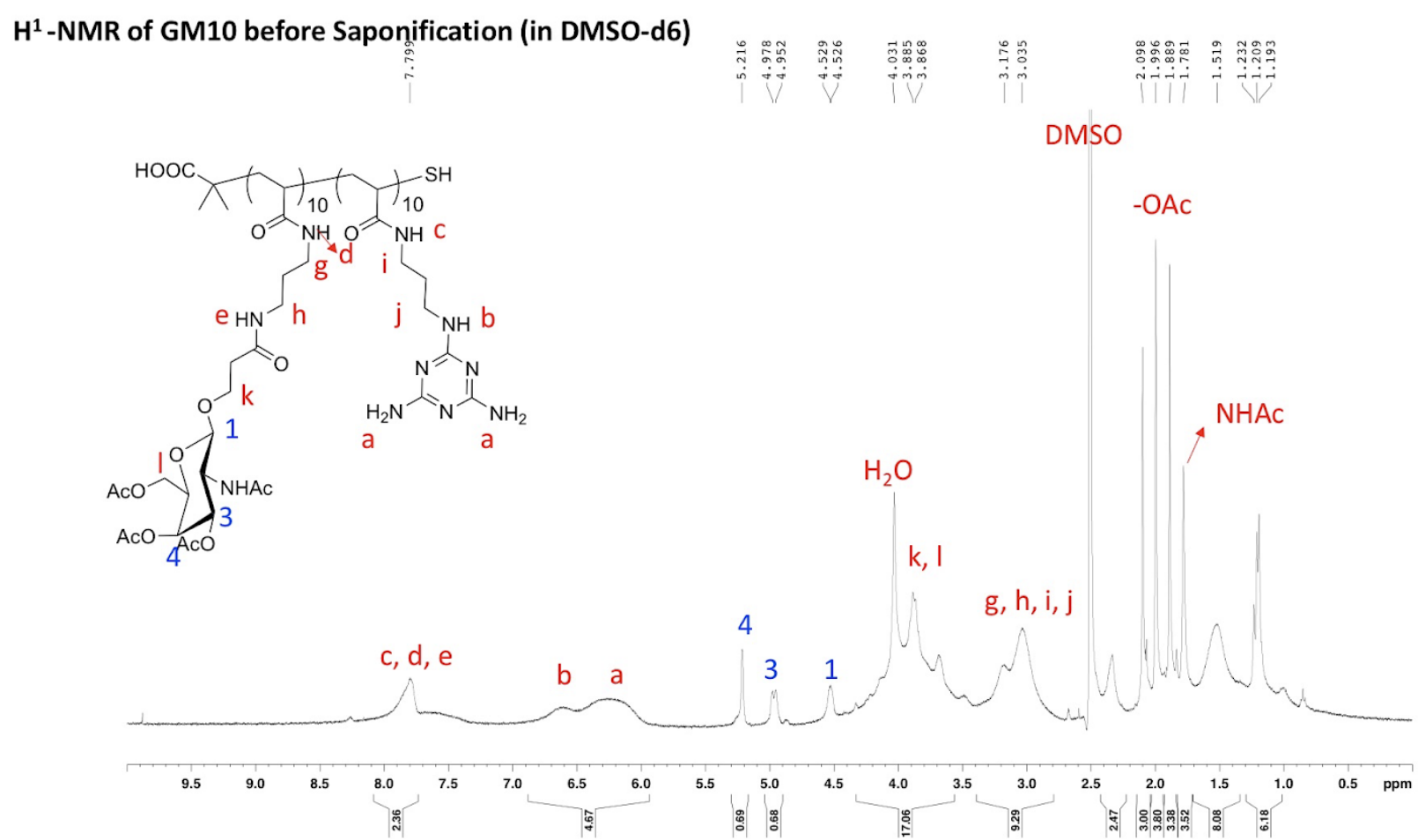

Figure S7. H1-NMR Spectrum of GM10, prior to the saponification step. The assignments are made as indicated above. When compared with the spectrum of GalNAc-NHS (Figure S6), most of the signature peaks from the sugar ring $(1,3,4$ in blue), as well as the -OAc, -NHAc peaks are still present in the polymer spectrum, albeit being more broad. Meanwhile, the typical peaks from melamine ( $\mathbf{a}, \mathbf{b}$ in red) are also present in the polymer spectrum. It's therefore evident that both melamine and GaINAc are incorporated onto the polymer. 


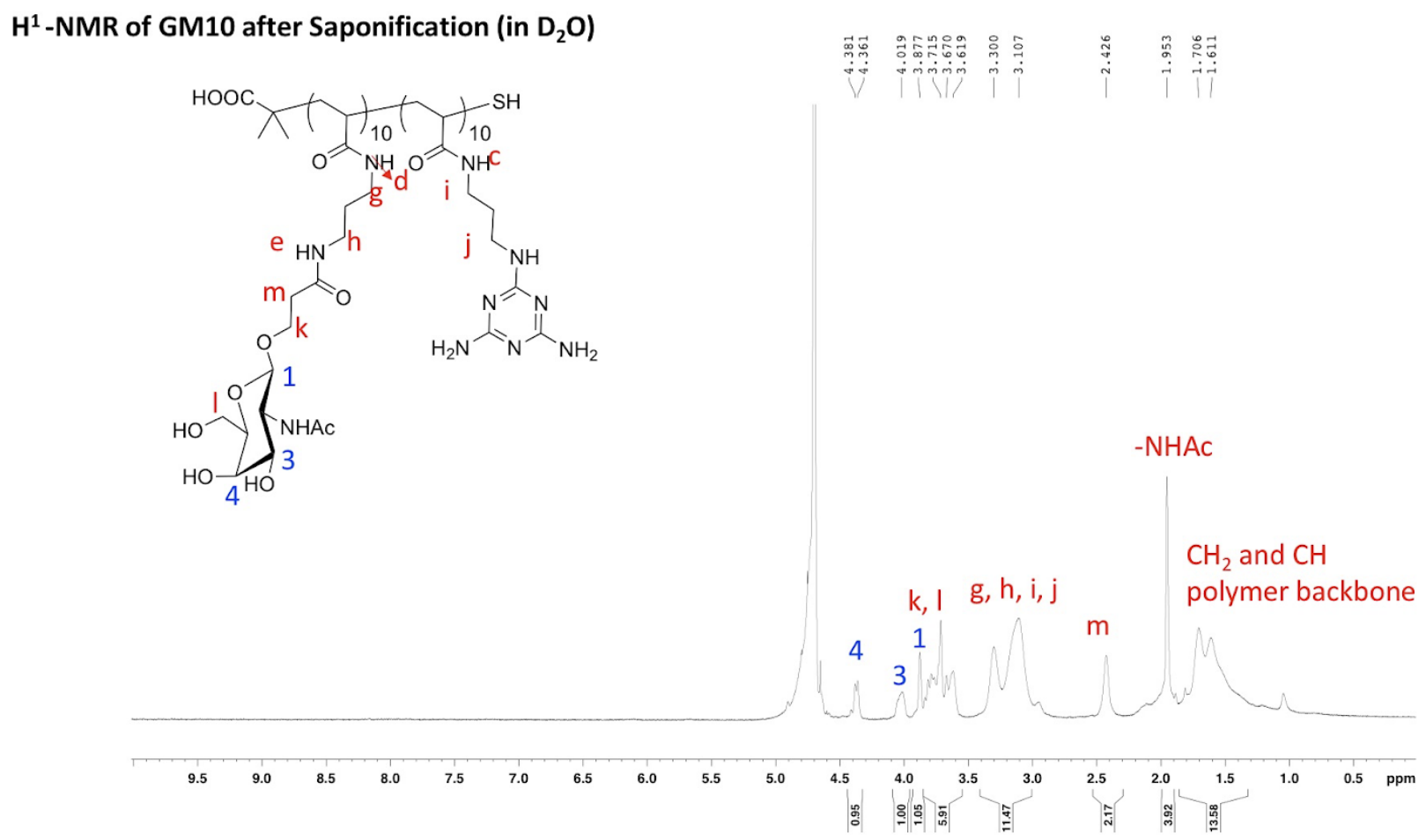

Figure S8. H1-NMR Spectrum of GM10, after the saponification step in $\mathrm{D}_{2} \mathrm{O}$ (deprotected $\mathrm{GM} 10$ can only dissolve in $\mathrm{D}_{2} \mathrm{O}$ ). The assignments are made as indicated above. When compared with the spectrum of GalNAc-NHS (Figure S4), most of the signature peaks from the sugar ring (1, 3, 4 in blue) are still present in the polymer spectrum, albeit being more broad. Also, due to the saponification, the three OAc peaks in Figures $\mathbf{S 6} \& \mathbf{S 7}$ are eliminated, but the -NHAc peak $(2.0 \mathrm{ppm})$ still remains. Due to exchange with deuterium, the peaks for melamine $\mathrm{NH}_{2}$ peaks are lost, but peaks i\&j remain.

\section{REFERENCES}

Zhou, J., Li, H., Li, S., Zaia, J., and Rossi, J.J. (2008). Novel Dual Inhibitory Function Aptamer-siRNA Delivery System for HIV-1 Therapy. Mol. Ther. 16, 1481-1489. 